\title{
Response letter to "What can be more prognostic than the pTNM category assessed in radical cystectomy samples?" by Sükösd F, Ivanyi B and Pajor $L$
}

\author{
Nadine T. Gaisa $^{1} \cdot$ Ruth Knüchel-Clarke ${ }^{1}$
}

Received: 9 August 2015 / Accepted: 13 August 2015 /Published online: 21 August 2015

(C) Springer-Verlag Berlin Heidelberg 2015

We thank Sükösd et al. for their critical judgement and discussion of our recent article [1]. However, several aspects they bring up evoke further discussion and require clarification. Sükösd et al. mainly criticize our use of a pTsum category (= sum of tumour stage of cystectomy and previous TUR-B specimen) since they are concerned that this might bias the pTstage. We respectfully disagree with this point of view, as in our article, the two distinct major aspects should not be mixed. The first concerns the definition of prognostically significant parameters (using pTsum). The second addresses a comparison of embedding protocols to be used to derive these parameters (in which only pTmax. of the cystectomy specimen is used).

Regarding the definition of prognostically significant parameters, we consider the tumour as a single biological event, even if part of the tumour was resected by TUR-B prior to cystectomy. In our clinic, non-muscle invasive bladder cancer patients (NMIBC $<$ pT2) are subjected to a second TUR-B up to 6 weeks after the first one when the tumour is high grade, pT1, large or incompletely resected (European Association of Urology (EAU) guidelines for NMIBC; [2]). Taking latency into account, we consider TUR-B specimens up to 3 months prior to cystectomy with the cystectomy specimen as one single tumour event. In our perception, prognostic parameters are provided by this tumour event and should not be limited to

Ruth Knüchel-Clarke

rknuechel-clarke@ukaachen.de

1 Institute of Pathology, RWTH Aachen University, Aachen, Germany tumour residues in the cystectomy specimen only. The bias of missing information in our study is as low as possible, since we reviewed all available TUR-B specimens, also from external pathology centres. Statistical calculation of (T stage) numbers only does not reflect the biology of the tumour. This justifies the use of pTsum for our Kaplan-Meier calculations to define significant parameters.

Correct indications for cystectomy are stage $\geq \mathrm{pT} 1$, high grade, recurrent or concurrent CIS, aggressive subtype (e.g. micropapillary variant), massive tumour load or multiple tumours and failure of conservative treatment (EAU guidelines for NMIBC; [2]). Following the EAU guidelines, a cystectomy cohort results mainly in higher pT stages and this might explain why the proportion of pT2 and pT3 tumours in our cohort were higher compared to those presented by Sükösd et al.. In addition, as stated in the "Materials and methods" section, we completely embedded the prostate (EAU guidelines for prostate cancer; [3]) in order to assure the detection of any pT4 stage. Conceivably, the higher proportion of pT4 stages in Sükösd et al. might be due to regional differences, differences in cohort inclusion criteria and different therapeutic approaches/surgery indications.

Concerning the comparison of embedding protocols, we have to resolve a misunderstanding. The comparison is based on the pTmax. of cystectomy specimens only. This is explained in Table 5 and Online resource 2. Indeed, the use of pTsum would not be justified here.

Finally, while we agree with Sükösd et al. that whole specimen embedding is more precise, our study shows that whole specimen embedding is not superior regarding $\mathrm{pT}$ and $\mathrm{V}$, the most relevant parameters for prognosis in bladder cancer. Our paper presents the results of a single-centre cohort only, which has its limitations. However, based on our data, we do not require whole-organ embedding of cystectomy specimens for diagnostic and prognostic reasons. 


\section{References}

1. Gaisa NT, Wilms H, Wild PJ, Jakse G, Heidenreich A, Knuechel R (2015) In cystectomy specimens with bladder cancer whole organ embedding increases the detection rate of histopathological parameters, but not of those with prognostic significance. Virchows Arch 466:423-432

2. Babjuk M, Burger M, Zigeuner R, Shariat SF, van Rhijn BW, Comperat E, Sylvester RJ, Kaasinen E, Böhle A, Palou Redorta J,
Roupet M (2013) European Association of Urology. EAU guidelines on non-muscle-invasive urothelial carcinoma of the bladder: update 2013. Eur Urol 64:639-653

3. Mottet N, Bastian PJ, Bellmunt J, van den Bergh RCN, Bolla M, van Casteren NJ, Cornford P, Joniau S, Mason MD, Matveev V, van der Kwast TH, van der Poel H, Rouviere O, Wiegel T (2014) Guidelines on prostate cancer. Update March 2015. European Association of Urology 2014. www.uroweb.org/ wp-content/uploads/09-Prostate-Cancer_LR.pdf 